\title{
Quaderni
}

QUADERNI Communication, technologies, pouvoir

\section{Nicolas Kaciaf, Les pages « Politique ». Histoire du journalisme politique dans la presse française (1945-2000)}

\section{Antoine Machut}

\section{(2) OpenEdition Journals \\ Édition électronique \\ URL : http://journals.openedition.org/quaderni/1085 \\ DOI : 10.4000/quaderni.1085 \\ ISSN : 2105-2956 \\ Éditeur \\ Les éditions de la Maison des sciences de l'Homme}

Édition imprimée

Date de publication : 5 mai 2017

Pagination : 99-105

Référence électronique

Antoine Machut, « Nicolas Kaciaf, Les pages « Politique ». Histoire du journalisme politique dans la presse française (1945-2000) », Quaderni [En ligne], 93 | Printemps 2017, mis en ligne le 05 mai 2017, consulté le 10 décembre 2020. URL : http://journals.openedition.org/quaderni/1085 ; DOI : https:// doi.org/10.4000/quaderni.1085 


\section{Compte rendu}

\section{Les pages " Politique ». Histoire du journalisme politique dans la presse française (1945-2000) Nicolas Kaciaf}

Rennes, Presses universitaires de Rennes, collection « Res Publica », 2013

par Antoine Machut Sciences Po Grenoble - PACTE

1. Sandrine Lévêque, Les journalistes sociaux. Histoire et sociologie d'une spécialité journalistique, Rennes, Presses Universitaires de Rennes, 2000.
Le livre de Nicolas Kaciaf prend pour objet une forme du journalisme dont seule l'élite visible avait suscité l'attention des chercheurs en sciences sociales. Comme Sandrine Lévêque ${ }^{1}$ l'avait fait à propos des journalistes sociaux, il écrit une « socio-histoire » (p. 14) du journalisme politique, avec le parti pris original de centrer l'étude sur les pages politiques - combinées avec des entretiens - plutôt que sur des archives d'organisations professionnelles. Ce choix ne conduit pas à une simple analyse de contenu mâtinée de contextualisation historique. L'objectif va bien au-delà : il s'agit pour l'auteur de décrire et d'expliquer les mutations dans les pratiques des journalistes politiques de la presse écrite française de 1945 à 2006. Les rhétoriques des journalistes ne sont pas étudiées en tant que telles mais comme la réponse à des contraintes professionnelles, et la traduction de postures journalistiques qui relèvent d'un ajustement constant des relations avec les sources, les lecteurs, les confrères et la hiérarchie. À ce titre, ce livre peut donc avoir prétention à décrire et expliquer les mutations de ce que l'on pourrait appeler un monde de l'information politique comprenant, au-delà du champ journalistique, les pratiques des hommes politiques, celles des lecteurs et enfin les configurations sociohistoriques dans lesquelles ils sont tous engagés.

Le récit historique de l'auteur repose essentiellement sur deux types de matériaux. Il s'agit d'abord de l'analyse qualitative fine des pages politiques - aussi bien le contenu des articles que la maquette des journaux - de six quotidiens (Le Figaro, France-Soir, L'Humanité, Libération, Le Monde, Paris-Presse) et cinq hebdomadaires français (L'Évènement du Jeudi, 
L'Express, France Observateur - Le Nouvel Observateur, Marianne, Le Point) sur une période allant de 1945 à 2006. Le choix de ces journaux est justifié par l'objectif de couvrir un spectre le plus large possible du point de vue du positionnement politique des journaux et du lectorat qu'ils visent, sans laisser de côté les titres de presse qui ont fini par disparaître, et ne raconter que «l'anachronique histoire des vainqueurs » (p. 15). Il s'agit ensuite du recours aux entretiens menés avec 65 journalistes qui ont participé à l'histoire du journalisme politique. D'un point de vue idéal-typique, on peut dire que Nicolas Kaciaf retrace le déplacement des pages politiques sur un continuum qui va d'une sténographie de l'activité parlementaire sous la IV République à la diversification et la personnalisation de l'information politique, valorisant désormais le spectacle de la compétition politique. Le récit de cette intrigue forme les deux premières parties du livre : la description du journalisme « parlementaire » d'abord, celle de sa métamorphose ensuite. L'élucidation des conditions (changements socio-politiques, reconfiguration de l'espace médiatique et renouvellement générationnel des journalistes) qui ont rendu possible cette métamorphose forme la troisième partie.

Nicolas Kaciaf commence par préciser que les forces du marché se manifestent dans le champ du journalisme bien avant les décennies 1970 et 1980. Le foisonnement de la presse au sortir de la Seconde Guerre mondiale, qui se voulait le moteur de la reconstruction morale du pays, est une parenthèse dans l'histoire de la presse, vite rattrapée par le processus de concentration du marché et l'affaiblissement des journaux les plus militants. Dès 1947, les pratiques d'avant-guerre en matière d'information politique sont reprises, ajustées au régime parlementaire de la $I V^{\mathrm{e}}$ République et aux contraintes quotidiennes de la production journalistique. Trois caractéristiques peuvent être considérées comme typiques de ces pratiques, majoritaires jusqu'à la fin des années 1950. En premier lieu, l'information politique n'est pas rapportée dans une rubrique « politique » spécifique mais souvent dispersée dans les pages des journaux, à l'exception du Monde et de Paris-Presse qui disposent déjà d'un espace dédié. En second lieu, le modèle de division du travail globalement partagé par les rédactions à cette époque est marqué par la prépondérance d'un « journalisme d'enregistrement » consistant à recueillir les déclarations politiques officielles et officieuses, auxquelles seuls les chefs du service " Politique » apportent un commentaire signé. Enfin, les journalistes « parlementaires » tirent leur réputation d'objectivité du caractère officiel de l'information politique sans que cela ne les empêche de s'inves- 
2. Ce penchant mendésiste, dans des années d'aprèsguerre jusqu'en 1960 où les hauts fonctionnaires constituent des sources prestigieuses alors que l'État n'a guère de difficultés à réguler la vie économique et sociale du pays a aussi été relevé chez les journalistes économiques par Philippe Riutort, «Le journalisme au service de l'économie », Actes de la recherche en sciences sociales $\mathrm{n}^{\circ} 1$, vol. 131, 2000, pp. 41-55.

3. Le concept de " cadrage » désigne ici la façon dont les médias participent à définir une thématique et ce qu'elle a de problématique en mettant l'accent sur certains de ses aspects au détriment d'autres. tir dans l'affrontement politique, valorisant des « hommes providentiels » susceptibles d'arbitrer les conflits politiques, en particulier Pierre MendèsFrance ${ }^{2}$.

Garantie d'impartialité, le « journalisme d'enregistrement » va progressivement devenir un handicap pour la presse écrite. Pour résumer la dense description réalisée par l'auteur, la trame de la métamorphose des pages politiques peut être rapportée en trois temps. Il s'agit d'abord d'un changement stylistique qui voit s'effacer les comptes rendus exhaustifs de séance au profit d'articles plus synthétiques, qui traitent sur le mode du récit l'information obtenue dans les « coulisses » du parlement. Les journalistes rompent peu à peu avec les formes du journalisme d'enregistrement, lui préférant des papiers anglés, contextualisés, où la parole des parlementaires sert désormais à illustrer l'angle retenu. Le style narratif et ses « effets de réel », importé initialement du monde anglo-saxon par la presse magazine en fort développement dans les années 1960, finit par supplanter la restitution froide des débats parlementaires dans la presse quotidienne nationale. À en croire un rédacteur en chef de L'Express, une des traditions de l'hebdomadaire dans les années 1980 était ainsi d'envoyer les stagiaires dans les restaurants parisiens recueillir le menu des hommes politiques...

Ce changement stylistique n'est pas une simple coquetterie de journaliste mais concrétise une modification du « cadrage ${ }^{3}$ » de l'information politique - c'est notre deuxième temps - qui décale le projecteur des institutions vers les personnalités politiques et valorise le «jeu» de la compétition plutôt que l'enjeu idéologique des questions de société. La médiatisation du politique ne passe plus essentiellement par celle du Parlement, mais se porte de plus en plus sur les partis politiques et une constellation d'acteurs qui parcourent les coulisses des institutions : lobbies, cabinets ministériels, etc. Il s'agit désormais de dévoiler la face cachée des processus décisionnels. La réorganisation progressive des rédactions et des maquettes « routinise » ce nouveau cadrage autour d'un rubricage « partisan » : le départ d'un « couloiriste », chargé d'arpenter les coulisses du parlement pour Le Monde, est comblé au début des années 1960 par une recrue chargée de suivre des mouvements d'extrême droite. Cette mise en scène du spectacle de la compétition politique attire régulièrement des critiques aux journalistes politiques, qui se voient reprocher d'ignorer le fond des débats politiques. Mais le procès en superficialité fait aux journalistes « politiques » est, pour Nicolas Kaciaf, assez 
réducteur dans la mesure où « on pourrait suggérer que les lectures les plus personnalisées et cyniques du jeu politique contribuent tout autant à évacuer les enjeux sociaux sous-jacents aux luttes partisanes qu'à déconstruire les mythes démocratiques (...)» (p. 340).

De fait, les transformations du mode de traitement et d'écriture de l'information « politique » rend possible l'adoption de nouvelles conceptions du rôle journalistique et de l'excellence professionnelle, troisième marque des métamorphoses des pages politiques. La description de la vie politique comme une comédie humaine procède à la fois d'une proximité forte avec le personnel politique, dont on dépeint les traits psychologiques, et d'une distanciation par le recours fréquent à un ton ironique et désinvolte. Cette distanciation des journalistes politiques leur permet d'adopter une nouvelle rhétorique de l'impartialité et de se poser en connaisseurs des règles pragmatiques du jeu politique. Cette posture d'arbitre est en outre outillée par la production croissante de sondages, utiles aux trames narratives pour personnifier l' " opinion publique » et se détacher d'une actualité traditionnellement rythmée par des périodes d'élection et de repos. Elle est aussi une réponse aux impératifs de n'être ni idéologues, ni complices passifs de la communication politique, selon les attentes perçues par les journaux chez un lectorat composé majoritairement de cadres, cible privilégiée depuis les années 1970 car la plus intéressante commercialement. Comme le remarque l'auteur, rien ne permet néanmoins d'affirmer que le traitement plus personnalisé de la politique a permis de conquérir de nouveaux lecteurs. Mais il offre en revanche un support aux journalistes pour affirmer leur compétence auprès de leurs sources et de leurs confrères, en montrant l'étendue de leur réseau ou en attestant qu'ils « connaissent le milieu ». Tirant leur autorité professionnelle de leur capacité à raconter et commenter le pouvoir et ses tactiques, les journalistes politiques ont laissé le genre « investigation » et la révélation de scandales politico-financiers ${ }^{4}$ se développer dans les services d'information générale de certains journaux, tandis que les journalistes « Société » prenaient en charge dans certaines rédactions la description des conséquences sociales de la politique sur les « vraies gens ».

Sans réduire cette métamorphose à une série de ruptures clairement situées historiquement ou l'imputer à des facteurs nettement identifiables, l'auteur rapporte enfin les conditions qui l'ont progressivement rendue possible. Il s'agit d'abord du changement dans les modes de gouvernement politique. 
La bipolarisation de la vie politique encouragée par le passage à la $\mathrm{V}^{\mathrm{e}}$ République a rendu plus acceptable commercialement et professionnellement le traitement médiatique approfondi des enjeux partisans. Elle ouvre aussi la voie à une génération de journalistes que la guerre d'Algérie a rendus plus critiques de la IV $\mathrm{V}^{\mathrm{e}}$ République, et plus enclins à s'engager idéologiquement dans une époque contestataire. À l'inverse, les alternances gouvernementales des années 1980 rendent commercialement plus risqués les engagements idéologiques fermes, et encouragent au contraire un positionnement nonmilitant, désenchanté et désinvolte. Peu à peu, les journalistes délaissent les institutions parlementaires non par clairvoyance, mais plus pragmatiquement parce que leur vivier de sources s'y assèche, tandis qu'arrive dans d'autres lieux une nouvelle génération d'hommes politiques qui s'ajustent aux nouveaux impératifs de communication, s'adaptant volontiers aux logiques journalistiques de vulgarisation. Le journalisme d'institution devient plus coûteux à défendre, et est abandonné au nom d'un principe de réalisme qui prend acte du désenchantement supposé des lecteurs pour la politique comme débats d'idées. Cette évolution ne s'appuie pas tant sur la dépolitisation des journalistes que sur l'affaiblissement de l'autorité symbolique des hommes politiques. Les convictions politiques des journalistes sont simplement mises en retrait par la nouvelle contrainte professionnelle de distanciation d'une part, et par le fait que la modération de la ligne éditoriale dans la plupart des journaux apaise les conflits idéologiques qui pouvaient autrefois éclater entre les journalistes et leur direction d'autre part.

Les changements de style rhétorique dans le traitement de l'information politique sont aussi une réponse à la crise commerciale des quotidiens, dont la baisse de la diffusion à partir des années 1970 contraste avec le succès des newsmagazines naissants. Les stratégies adoptées sont variées, en fonction du style et du ton historiques de chaque journal, mais la logique commerciale - véhiculée par des spécialistes du marketing de plus en plus présents dans les salles de rédaction - prend globalement le dessus et commande de produire des contenus moins ennuyeux. Elle incite aussi les directeurs de journaux à nommer comme chefs de service des journalistes réputés peu vigoureux idéologiquement, et à métisser politiquement leur rédaction, non sans susciter des conflits. Le Monde a ainsi attribué la chute de sa diffusion au début des années 1980 à une trop grande indulgence vis-à-vis d'un pouvoir socialiste impliqué dans divers scandales (dont celui du Rainbow Warrior). 
Le traitement diversifié, personnalisé et analytique de l'information politique a enfin également trouvé son impulsion dans le renouvellement générationnel des journalistes. L'auteur montre en particulier comment la montée du niveau de diplôme des recrutés et la relative féminisation du journalisme politique se sont articulées avec les nouvelles exigences rédactionnelles de généralisation du décryptage et de mise en récit des personnalités politiques. Il recense finalement trois générations de journalistes politiques (respectivement " gaullienne », « de la bipolarisation » et « des alternances ») qui ont, par petites touches successives, contribué à renouveler les routines rédactionnelles.

C'est finalement le grand mérite de cet ouvrage que d'avoir su retracer de manière convaincante une métamorphose progressive, non-linéaire et contingente, en respectant les spécificités de chaque journal (globalement lissées dans ce compte-rendu, par souci de synthèse). Les nombreux indicateurs - simples et bien choisis -, les extraits d'entretiens et d'articles renforcent la démonstration autant qu'ils donnent de la chair aux évolutions observées. Commentant les nouvelles pratiques de narrations relevées dans les pages politiques, Nicolas Kaciaf a recours à une phrase de Paul Ricoeur qui aurait tout aussi bien pu être utilisée pour éclairer l'objectif atteint par son livre : " [L'intrigue] fait médiation entre des évènements ou des incidents individuels, et une histoire prise comme un tout. [...] Comprendre l'histoire, c'est comprendre comment et pourquoi les épisodes successifs ont conduit à cette conclusion, laquelle, loin d'être prévisible, doit être finalement acceptable » (cité pp. 186-187). C'est bien parce qu'il a su retracer son intrigue que l'auteur permet de l'histoire du journalisme politique.

Les critiques qui viennent à l'esprit au moment de pointer les limites de l'ouvrage sont alors peu nombreuses. Disons seulement que l'approche et la structuration de l'ouvrage produisent parfois des effets de répétition entre la description et l'explication, et rendent mal aisée la défense d'une thèse forte à propos du problème posé. Érik Neveu l'a remarqué dans sa préface, Nicolas Kaciaf « semble répugner à un engagement paradigmatique trop précis » (p. 9). Pour autant, il prend part à des controverses théoriques passionnantes et ouvre des questionnements qui dépassent l'objet initial de sa recherche. Il en va ainsi lorsqu'il réhabilite - sans toutefois le dire explicitement l'analyse goffmanienne des cadres, faisant du cadrage « stratégique » de la politique une «réponse pratique » (p. 294) à des contraintes situationnelles 
5. Erving Goffman, Les cadres de l'expérience. Paris, France, les Éditions de Minuit, 1991. La thèse de la spirale de cynisme a été développée dans Joseph N. Cappella et Kathleen Hall Jamieson, Spiral of Cynicism: The Press and the Public Good. 1 edition. New York: Oxford University Press, 1997. Pour une critique des analyses de cadres décorrélées des situations pratiques de " cadrage ", voir Cédric Terzi, «"Qu'avez-vous fait de l'argent des juifs?": problématisation et publicisation de la question "des fonds juifs et de l'or nazi" par la presse suisse, 1995-1998 ». Thèse de doctorat, Paris, France: Centre d'étude des mouvements sociaux, 2004, pp. 210-215. spécifiques plutôt que la résultante de dispositions mentales, en l'occurrence le cynisme grandissant des journalistes et de leur hiérarchie ${ }^{5}$. Le lecteur plus largement intéressé par les questions théoriques de sociologie des médias et du journalisme trouvera donc lui aussi matière à réflexion dans ce livre. 
